\title{
Synthesis of Nickel Nanoparticles using Electron Beam Irradiation
}

\author{
Seung Jun Lee, Hyun Bin Kim, Seung Hwan Oh, and Phil Hyun Kang* \\ Research Division for Industrial and Environment, Korea Atomic Energy Research Institute, 29 Geumgu-gil, \\ Jeongup-si, Jeollabuk-do 56212, Korea
}

(Received 23 December 2014, Received in final form 4 August 2015, Accepted 2 September 2015)

\begin{abstract}
A study on the preparation of nickel oxide nanoparticles using electron beam irradiation is described. Nickel nanoparticles were synthesized with nickel chloride hexahydrate as a metal precursor and different sodium hydroxide concentrations using electron beam irradiation. The effects of sodium hydroxide concentration and electron beam absorbed doses were investigated. The samples were synthesized at different sodium hydroxide concentrations and with absorbed doses of 100 to $500 \mathrm{kGy}$ at room temperature. Synthesized nanoparticles were characterized by X-ray diffraction (XRD) and field emission scanning electron microscopy (FE-SEM) and a vibrating sample magnetometer (VSM). The nanoparticle morphologies seemed to be non-spherical and aggregated. The 1:1 molar ratio of nickel chloride hexahydrate and sodium hydroxide showed a higher purity and saturation magnetization value of $13.0 \mathrm{emu} / \mathrm{g}$. The electron beam absorbed dose was increased with increasing nickel nanoparticle nucleation.
\end{abstract}

Keywords : nickel nanoparticles, magnetic materials, electron beam

\section{Introduction}

In recent years, magnetic nanoparticles are used in many applications including a magnetic fluid, data storage and catalysts, contrast enhancement in magnetic resonance imaging, magnetic carriers, and biomedicine because of their physical and magnetic properties [1].

Nickel nanoparticles have also been investigated because of their magnetic properties. Nickel nanoparticles have produced many methods in the literature such as electrical, chemical reduction, sol-gel reaction [2], micro emulsion [3], thermal decomposition, hydrothermal [4], reducing agent using [5-7], and polyol process. Although there are various synthesis methods of nickel nanoparticles, thermal decomposition is a conventional method used to produce stable monodispersed nanoparticles [8]. Each of these processes requires heating and a long reaction time for the catalyst addition. Therefore, the materials were limited to use as a precursor for synthesis of nanoparticles. An electron beam irradiation method can be produced under easy and fast room-temperature processing without a catalyst or thermal process. The

(C)The Korean Magnetics Society. All rights reserved.

*Corresponding author: Tel: +82-63-570-3061

Fax: +82-63-570-3068, e-mail: phkang@kaeri.re.kr synthesis of metal nanoparticles using radiation is a promising substitution because of a high reduction potential. An electron beam irradiation method used to synthesize metal nanoparticles has been reported with silver, copper, and gold nanoparticles, among others [9-11].

In our study, we synthesized nickel nanoparticles using the electron beam irradiation of nickel chloride hexahydrate precursor in DI water at room temperature. The synthesized $\mathrm{Ni}$ nanoparticles were studied at different additions of sodium hydroxide concentration and electron beam doses. Morphology and particle size were studied using field emission scanning microscopy (FE-SEM). Structure was determined using X-ray diffraction (XRD). Magnetic properties were characterized using a vibrating sample magnetometer (VSM).

\section{Experimental}

Nickel hexahydrate $\left(\mathrm{NiCl}_{2} \cdot 6 \mathrm{H}_{2} \mathrm{O}\right)$ was purchased from Sigma-Aldrich, and sodium hydroxide solution $(1 \mathrm{~N} \mathrm{NaOH})$ was purchased from Showa chemical. All chemicals were used without further purification.

For the first sample series (a), the nickel hexahydrate $(0.1 \mathrm{~g})$ was dissolved in DI water $(10 \mathrm{ml})$. Four kinds of precursors were prepared by sodium hydroxide addition to achieve nickel to sodium ion ratios of 1:0.5, 1:0.75, 1:1 
Table 1. Experimental conditions to fabricate the precursors and electron beam irradiations.

\begin{tabular}{cccccc}
\hline \hline \multirow{2}{*}{ Series } & \multicolumn{3}{c}{ Precursor } & \multirow{2}{*}{$\begin{array}{c}\text { Electron Beam } \\
\text { Irradiation Dose }\end{array}$} \\
\cline { 2 - 5 } & $\mathrm{NiCl}_{2} \cdot 6 \mathrm{H}_{2} \mathrm{O}$ & $\mathrm{NaOH}$ & DI water & IPA & $300 \mathrm{kGy}$ \\
(a) & $0.1(\mathrm{~g})$ & $0.14,0.28,0.42,0.56(\mathrm{ml})$ & $10(\mathrm{ml})$ & $0.3(\mathrm{ml})$ & $100,200,300,500 \mathrm{kGy}$ \\
(b) & $0.1(\mathrm{~g})$ & $0.42(\mathrm{ml})$ & $10(\mathrm{ml})$ & $0.3(\mathrm{ml})$ & $100,200,300,500 \mathrm{kGy}$ \\
(c) & $0.1(\mathrm{~g})$ & $0.56(\mathrm{ml})$ & $10(\mathrm{ml})$ & $0.3(\mathrm{ml})$ & 100,300 \\
\hline
\end{tabular}

and 1:1.25, respectively. Isopropyl alcohol $(0.3 \mathrm{ml})$ was added into each precursor as a radical scavenger [10, 12]. Electron beam irradiation (300 kGy) was carried out by an electron beam accelerator (UELV-10-10S, beam energy: $10 \mathrm{MeV}$, current: $0.5 \mathrm{~mA}$ ) on each precursor at the Korea Atomic Energy Research Institute in Korea. The precipitated particles were washed with distilled water, separated by centrifugation three times, and then dried in a vacuum drying oven at room temperature.

Precursors for the sample series (b) and (c) were prepared using $0.42 \mathrm{ml}$ and $0.56 \mathrm{ml}$ of $1 \mathrm{~N} \mathrm{NaOH}$. In addition, electron beam irradiations of 100 to $500 \mathrm{kGy}$ were carried out. The other processes are the same with series (a). The sample series are summarized in Table 1.

Crystal structure of the nanoparticles was studied by X-ray diffraction (X'pert Powder, PANalytical) with $\mathrm{Cu} \mathrm{K} \alpha$ radiation $(\lambda=1.5606 \AA)$. Magnetic properties were characterized by a vibrating sample magnetometer (VSM, Lakeshore) at room temperature. Morphology of the nanoparticles was observed by field emission scanning electron microscope (S-4800, HITACHI) with an accelerating voltage of $15 \mathrm{kV}$.

\section{Results and Discussion}

\subsection{Crystal structure of nanoparticles}

An electron beam (300 kGy) was irradiated on each precursor prepared with different $\mathrm{NaOH}$ concentrations. It was found that black precipitates were formed in each sample after electron beam irradiation. When electron beam was irradiated on the samples, the hydrated electrons $\left(e_{e q}^{-}\right)$, hydroxyl radicals $\left(\mathrm{OH}^{*}\right)$, and $\mathrm{H}^{*}$ atoms were produced by irradiation through the following chemical reactions:

$$
\mathrm{H}_{2} \mathrm{O} \stackrel{\text { EB Irradiation }}{\longrightarrow} e_{e q}^{-}, \mathrm{H}_{3} \mathrm{O}^{+}, \mathrm{H}^{*}, \mathrm{H}_{2}, \mathrm{OH}^{*}, \mathrm{H}_{2} \mathrm{O}_{2}
$$

Nickel ions could be reduced into a zero-valent state by hydrated electrons $\left(e_{e q}^{-}\right)$and $\mathrm{H}^{*}$ atoms.

$$
\begin{aligned}
& \mathrm{Ni}^{2+}+2 e_{e q}^{-} \rightarrow \mathrm{Ni}^{0} \\
& \mathrm{Ni}^{2+}+2 \mathrm{H}^{\cdot} \rightarrow \mathrm{Ni}^{0}+2 \mathrm{H}^{+}
\end{aligned}
$$

The other way to reduce the nickel ions is using a radical scavenger, such as primary or secondary alcohols. It can be described for use of isopropyl alcohol in this study through the following reaction.

$$
\begin{aligned}
& \mathrm{OH}^{\bullet}+\mathrm{CH}_{2} \mathrm{CHOHCH}_{3} \rightarrow \mathrm{H}_{2} \mathrm{O}+\mathrm{H}_{3} \mathrm{CC}^{\bullet} \mathrm{OHCH}_{3} \\
& \mathrm{H}^{\bullet}+\mathrm{CH}_{2} \mathrm{CHOHCH}_{3} \rightarrow \mathrm{H}_{2}+\mathrm{H}_{3} \mathrm{CC}^{\bullet} \mathrm{OHCH}_{3} \\
& \mathrm{Ni}^{2+}+\mathrm{H}_{3} \mathrm{CC}^{\bullet} \mathrm{OHCH}_{3} \rightarrow \mathrm{CH}_{2} \mathrm{COCH}_{3}+\mathrm{Ni}^{0}+\mathrm{H}^{+}
\end{aligned}
$$

To determine the phase characterization of the nickel nanoparticles, we conducted XRD measurements. The XRD profiles of the precipitates are shown in Fig. 1 as a function of the $\mathrm{NaOH}$ addition. The broad peak over the $2 \theta=5-25^{\circ}$ results from the amorphous state. Two kinds of phase peaks were shown. One is a face-centered cubic (fcc) nickel phase showing characteristic peaks at $2 \theta$ values of $44.5^{\circ}, 51.8^{\circ}, 76.4^{\circ}$ corresponding to the indices of (111), (200) and (220). The other is $\mathrm{Ni}(\mathrm{OH})_{2}$ peaks at $2 \theta$ of $32^{\circ}, 38^{\circ}, 58^{\circ}, 62^{\circ}$ corresponding to (100), (101), (110) and (111). Both peaks increased with an increase in $\mathrm{NaOH}$ addition. This indicates that $\mathrm{NaOH}$ has an important role in producing nickel nanoparticles. Among previously mentioned chemical reactions, $\mathrm{OH}$ relates to reaction equation (4).

Two peaks in an amorphous band were estimated to

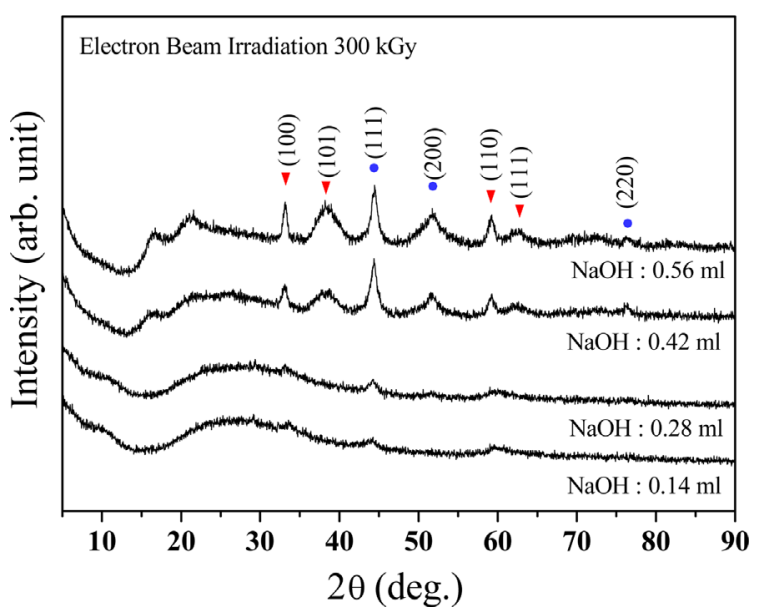

Fig. 1. (Color online) The XRD patterns of synthesized nickel nanoparticles with the addition of various $1 \mathrm{~N} \mathrm{NaOH}$ concentrations with an absorbed dose of $300 \mathrm{kGy}$. 


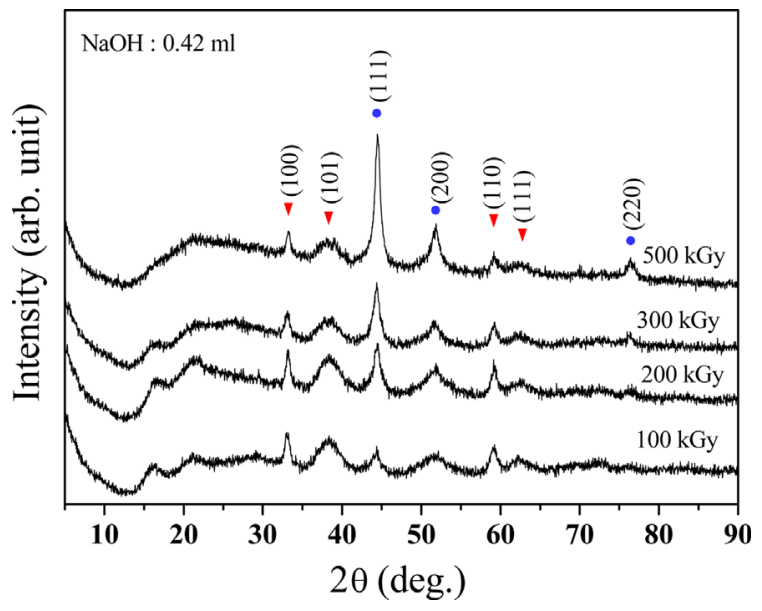

Fig. 2. (Color online) The XRD patterns of synthesized nickel nanoparticles with the addition of $0.42 \mathrm{ml}$ of $1 \mathrm{~N} \mathrm{NaOH}$ with various irradiation doses.

have a monoclinic $\mathrm{NaNiO}_{2}$ structure. Their peaks intensity decreased with an increase in the electron beam dose.

To confirm the role of $\mathrm{NaOH}$, the effects of electron beam irradiation dose was investigated. Figs. 2 and 3 show XRD profiles of 0.42 and $0.56 \mathrm{ml}$ of $1 \mathrm{~N} \mathrm{NaOH}$ added samples as a function of the electron beam irradiation dose. The electron beam penetrates into the solution, and then metal ions are reduced. $\mathrm{Ni}$ atoms are formed homogeneously everywhere within the solution. These generated atoms act as the centre of nucleation as a seed crystal. Particles growth could be determined by the collision of atoms or nuclei. A higher absorbed dose forms a cluster aggregation with metal nuclei, and ions are reduced on the seed crystal surface by a reducing agent [13]. The intensity of $\mathrm{Ni}(\mathrm{OH})_{2}$ is not changed by the electron beam irradiation dose in both sample series. In addition, the intensity of $\mathrm{Ni}(\mathrm{OH})_{2}$ shows a higher value at

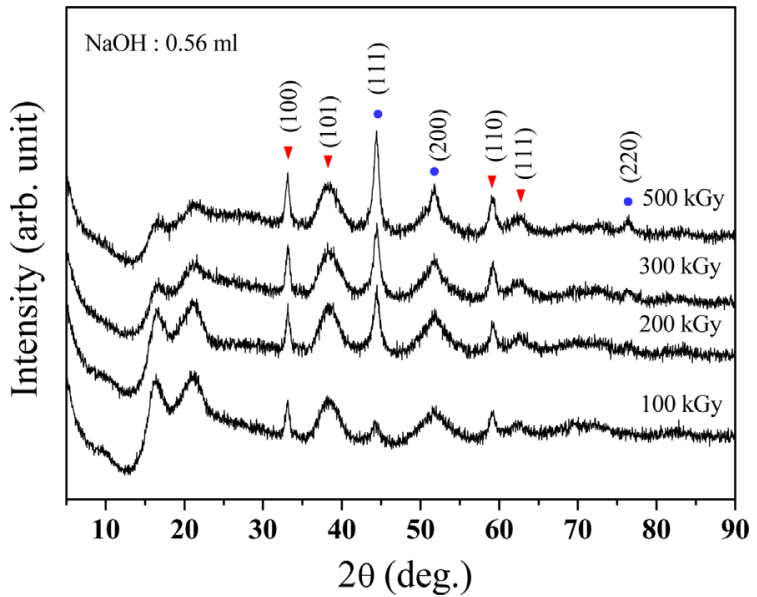

Fig. 3. (Color online) The XRD patterns of synthesized nickel nanoparticles with the addition of $0.56 \mathrm{ml}$ of $1 \mathrm{~N} \mathrm{NaOH}$ with various irradiation doses.

$0.56 \mathrm{ml}$ of the $1 \mathrm{~N} \mathrm{NaOH}$ added samples. This means that the formation of $\mathrm{Ni}(\mathrm{OH})_{2}$ only depends on the initial addition content of $\mathrm{NaOH}$. The intensity of the Ni peaks increase with increasing electron beam irradiation dose shown in Figs. 2 and 3.

\subsection{Morphology of nanoparticles}

The FE-SEM images of nanoparticles are shown in Fig. 4. Few nano-sized particles like aggregated dust were observed in the $500 \mathrm{kGy}$ electron beam irradiated samples. The nanoparticles were aggregated because of their high surface energy for the drying process. The size of the nanoparticles is about $20-30 \mathrm{~nm}$.

The average particle size of $\mathrm{Ni}$ is evaluated from the main peak following the Scherrer equation from the XRD profiles.

$$
t=(0.9 \lambda) /(B \cos \theta)
$$

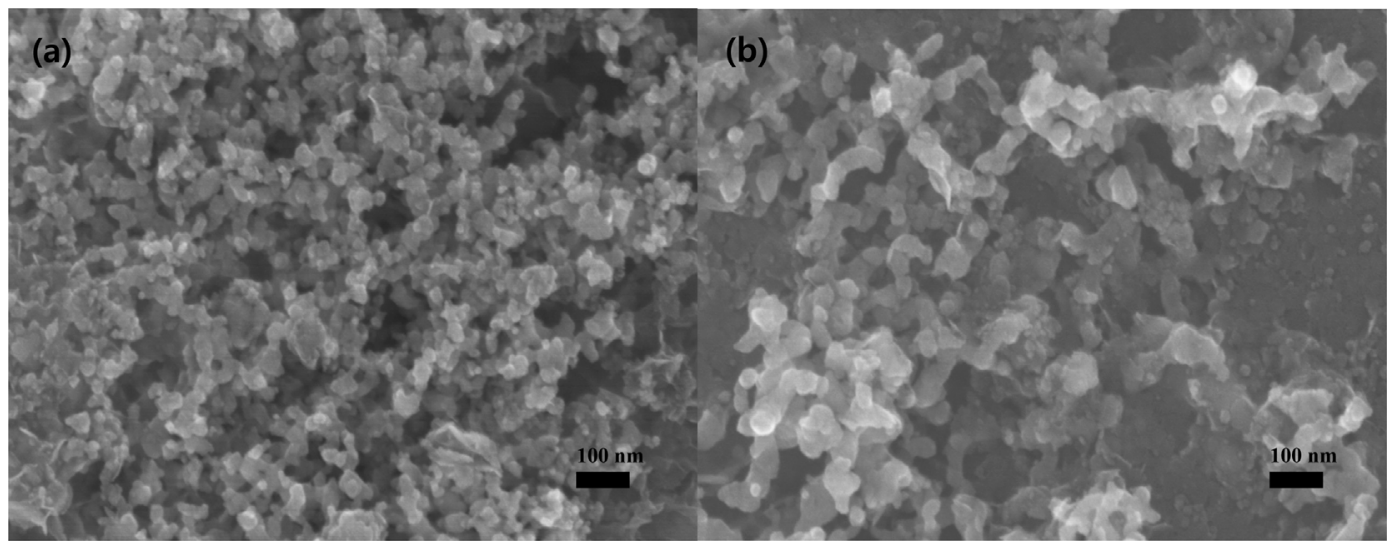

Fig. 4. FE-SEM images of nickel nanoparticles prepared by $500 \mathrm{kGy}$ using electron beam irradiation at a $1 \mathrm{~N} \mathrm{NaOH}$ concentration for (a) $0.42 \mathrm{ml}$ and (b) $0.56 \mathrm{ml}$. 

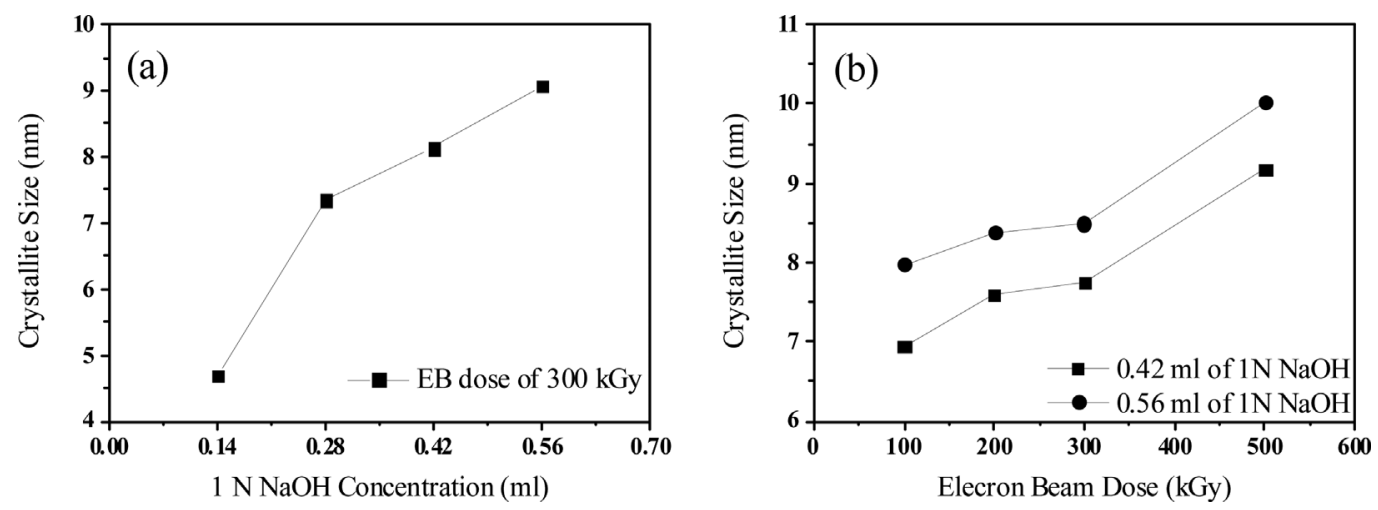

Fig. 5. Calculated mean crystalline sizes by the Scherrer equation: (a) EB dose of $300 \mathrm{kGy}$, (b) 0.42 and $0.52 \mathrm{ml}$ of $1 \mathrm{~N} \mathrm{NaOH}$.

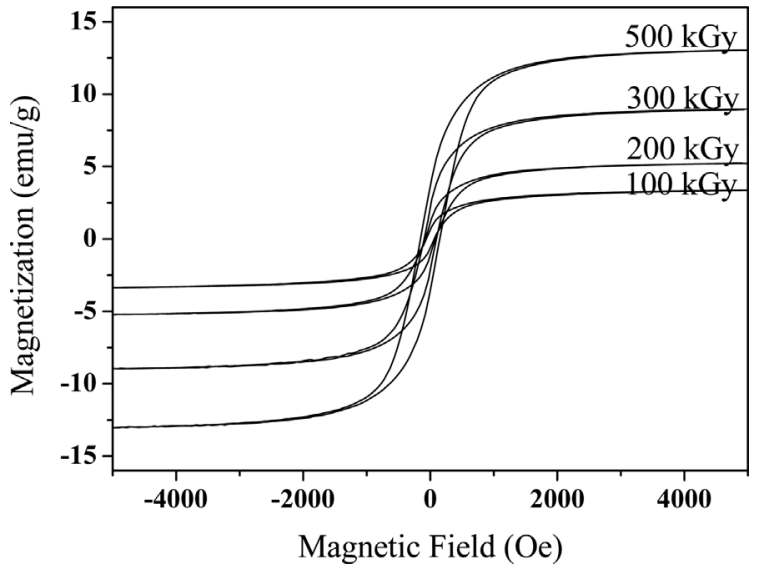

Fig. 6. Hysteresis loops of nickel nanoparticles in $0.42 \mathrm{ml}$ of a $1 \mathrm{~N} \mathrm{NaOH}$ added solution with electron beam doses of 100 to 500 kGy.

where $t$ is the mean crystallite size, $\lambda$ is the $\mathrm{X}$-ray wave length, $B$ is the full width at half maximum, $\theta$ is the Bragg angle, and a shape factor of 0.9 is used. The Ni

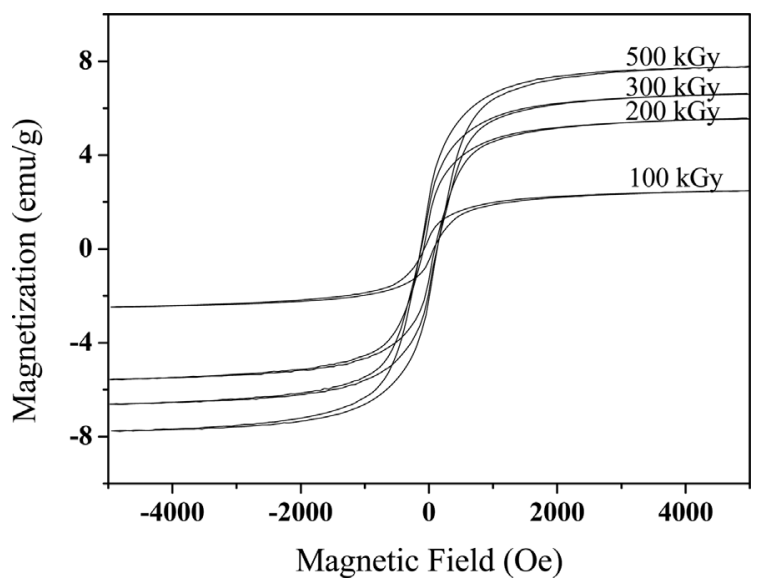

Fig. 7. Hysteresis loops of nickel nanoparticles in $0.56 \mathrm{ml}$ of a $1 \mathrm{~N} \mathrm{NaOH}$ added solution with electron beam doses of 100 to $500 \mathrm{kGy}$. crystallite size by the Scherrer Eq. is shown in Fig. 5. The mean crystallite size is smaller than in the SEM images. This indicates that the nanoparticles are composed of $\mathrm{Ni}$ and $\mathrm{Ni}(\mathrm{OH})_{2}$ phases. Compared to Figs. 4 and 5, these results suggest that nanoparticles are formed into several crystallites.

\subsection{The magnetic properties of nanopowders}

The magnetic properties were measured on a VSM at room temperature. The hysteresis loops are shown as a function of the electron beam doses in Figs. 6 and 7.

The obtained nanoparticles show a ferromagnetic behavior. The saturation magnetization $(M s)$ values are determined from the hysteresis loops at $5 \mathrm{kOe}$, as shown in Fig. 8.

Both $100 \mathrm{kGy}$ irradiated samples have similar $M s$ values. When increasing the irradiation dose, $M s$ also increases. The $M s$ values of series (b) and (c) at $500 \mathrm{kGy}$ reach $13 \mathrm{emu} / \mathrm{g}$ and $7 \mathrm{emu} / \mathrm{g}$, respectively. This result is $24 \%$ and $13 \%$ of the bulk Ni value of $54.39 \mathrm{emu} / \mathrm{g}$. The Ms decreased due to the oxidation phase of $\mathrm{Ni}$. In

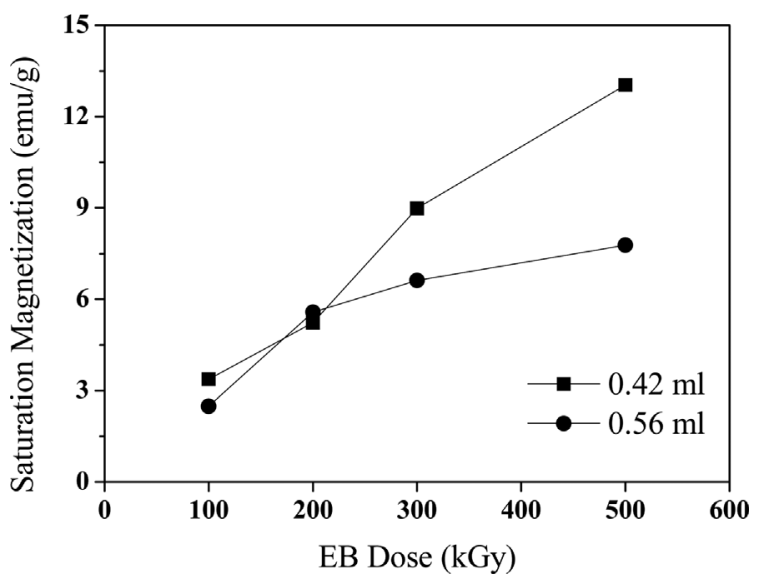

Fig. 8. Saturation magnetization values of absorbed doses of 100 to $500 \mathrm{kGy}$ with the addition of $1 \mathrm{~N} \mathrm{NaOH}$ concentrations. 


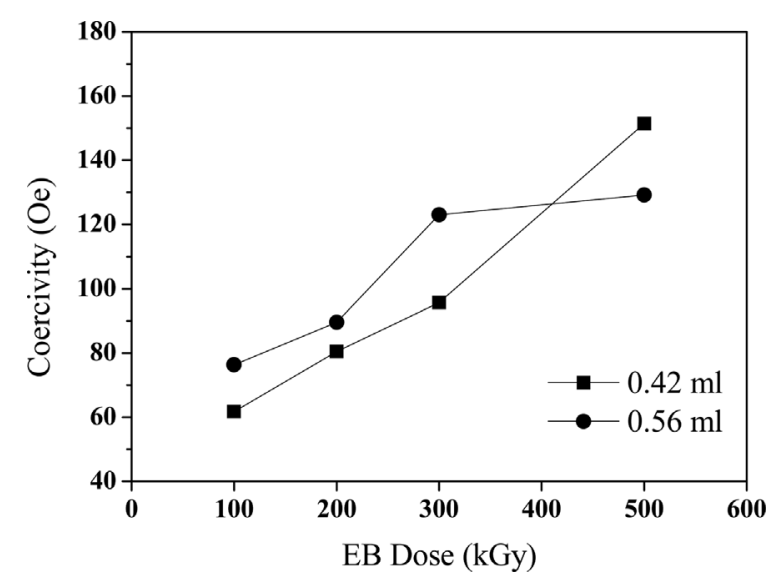

Fig. 9. Coercivity values of absorbed doses of 100 to 500 $\mathrm{kGy}$ with the addtion of $1 \mathrm{~N} \mathrm{NaOH}$ concentrations.

comparison with the XRD results, it matches the peak intensity ratio change of the $\mathrm{Ni}$ and $\mathrm{Ni}(\mathrm{OH})_{2}$ phases. The coercivities $(H c)$ also increased with an increase in the electron beam dose in Fig. 9.

The coercivity is related to the particle size. $H c$ increases with an increasing particle size until reaching the critical size of $21.2 \mathrm{~nm}$ for a single domain [14]. Hc decreases with an increasing size above the critical size. A synthesized nanoparticle is composed of $\mathrm{Ni}$ and $\mathrm{Ni}(\mathrm{OH})_{2}$ domains, as described in the XRD results. In our work, the $H c$ value increases with an increasing electron beam dose. These results were estimated that the domain size of synthesized $\mathrm{Ni}$ is smaller than the critical size.

\section{Conclusions}

Nickel nanoparticles have been synthesized with nickel chloride hexahydrate as a metal precursor and sodium hydroxide as a hydration agent through electron beam irradiation at room temperature without thermal decomposition. As a result, an optimized nickel chloride hexahydrate ratio of $1: 1$ with sodium hydroxide was the best condition in our case. The XRD patterns of the samples showed fcc nickel nanoparticles. The magnetic properties increased with an increase in the absorbed dose. The electron beam irradiation method is potentially a good candidate for synthesis of nickel nanoparticles.

\section{Acknowledgements}

This work was supported by the National Research Foundation of Korea (NRF) grant funded by the Korea government (MSIP) (No. 2012M2A2A6013183).

\section{References}

[1] H. Wang, X. Kou, L. Zhang, and J. Li, Mat. Res. Bull. 43, 3529 (2008).

[2] J. Gong, L. L. Wang, Y. Liu, J. H. Yang, and Z. G. Zong, J. Alloys Compd. 457, 6 (2008).

[3] A. Kumar, A. Saxena, A. De, R. Shnker, and S. Mozumdar, Adv. Nat. Sci. Nanosci. Nanotechnol. 4, 025009 (9pp) (2013).

[4] Z. Liu, S. Li, Y. Yang, S. Peng, Z. Hu, and Y. Qian, Adv. Mater. 15, 1946 (2003).

[5] Y. Hou and S. Gao, J. Mater. Chem. 13, 1510 (2003).

[6] M. Green and P. O'Brien, Chem. Commun. 1912 (2001).

[7] Y. Jeon, G. H. Lee, J. Park, B. Kim, and Y. Chang, J. Phys. Chem. B. 109, 12257 (2005).

[8] J. Park, E. Kang, S. U. Son, H. M. Park, M. K. Lee, J. Kim, K. W. Kim, H. J. Noh, J. H. Park, C. J. Bae, J. G. Park, and T. Hyeon, Adv. Mater. 17, 429 (2005).

[9] K. A. Bogle, S. D. Dhole, and V. N. Bhoraskar, Nanotechnology 17, 3204 (2006).

[10] L. Q. Pharm, J. H. Sohn, J. H. Park, H. S. Kang, B. C. Lee, and Y. S. Kang, Radiat. Phys. Chem. 80, 638 (2011).

[11] K. D. N. Vo, C. Kowandy, L. Dupont, X. Coqueret, and N. Q. Hien, Radiat. Phys. Chem. 94, 84 (2014).

[12] S. P. Ramnani, J. Biswal, and S. Sabharwal, Radiat. Phys. Chem. 76, 1290 (2007).

[13] A. Abedini, A. R. Daud, M. A. A. Hamid, N. K. Othman, and E. Saion, Nanoscale Res. Lett. 8, 474 (2013).

[14] H. T. Zhang, G. Wu, X. H. Chen, and X. G. Qiu, Mater. Res. Bull. 41, 495 (2006). 